\title{
CARRAPATOS DO GÊNERO AmblyOmma (ACARI: IXODIDAE) E SUAS RELAÇÕES COM OS HOSPEDEIROS EM ÁREA ENDÊMICA PARA FEBRE MACULOSA NO ESTADO DE SÃO PAULO
}

\author{
CARLOS ALBERTO PEREZ ${ }^{* 1}$; ÁLVARO FERNANDO DE ALMEIDA ${ }^{1}$; ALEXANDRE ALMEIDA²; VICTOR \\ HUGO BARBOSA DE CARVALHO ${ }^{3}$; DANIELE DO CARMO BALESTRIN ${ }^{1}$; MURILO SARAIVA GUIMARÃES ${ }^{3}$; \\ JULIO C. COSTA ${ }^{1}$; LEONARDO ADRIANO RAMOS ${ }^{1}$; ANA DULCE ARRUDA-SANTOS ${ }^{4}$; CLARICE PINTO \\ MÁXIMO-ESPÍNDOLA ${ }^{4}$; DARCI MORAES BARROS-BATTESTI ${ }^{4}$
}

\begin{abstract}
PEREZ, C.A.; ALMEIDA, A.F.DE; ALMEIDA, A.; CARVALHO, V.H.B.DE; BALESTRIN, D. DO C.; GUIMARÃES, M.S.; COSTA, J.C.; RAMOS, L.A.; ARRUDA-SANTOS, A.D.; MÁXIMO-ESPÍNDOLA, C.P.; BARROS-BATTESTI, D.M. [Ticks of genus Amblyomma (Acari: Ixodidae) and their relationship with hosts in endemic area for spotted fever in the state of São Paulo]. Carrapatos do gênero Amblyomma (Acari: Ixodidae) e suas relações com os hospedeiros em área endêmica para Febre Maculosa no estado de São Paulo. Revista Brasileira de Parasitologia Veterinaria v. 17, n. 4, p.210-217, 2008. Escola Superior de Agricultura Luiz de Queiroz, Av. Pádua Dias 11, CP 09, Piracicaba, SP 13418-900, Brasil. E-mail: c_aa_perez@hotmail.com

Seven species of mammals and 36 of birds were investigated to determine the tick prevalence and intensity of infestation. The study was conducted at the Esalq/USP in Piracicaba municipality, State of São Paulo. It was collected 52 mammals and 158 birds parasitized by 12,418 ticks. Adult ticks $(\mathrm{N}=7,343)$ were found on capybaras, while the immature were mainly collected on small mammals and birds. The main hosts for immatures in descending order were opossums (69.1\%), capybara (24.4\%) and black vultures (3.7\%). Among the avifauna, the black vulture (Cathartidae) had the heaviest infestation (69.9\%) followed by species of the Thamnophilidae and Turdidae families. Adult ticks collected on capybaras were A. cajennense (80.8\%) and A. dubitatum (19.2\%). Both tick species were also found on opossums corresponding to $72.4 \%$ and $27.6 \%$, respectively. Due to easy capture and attractiveness for ticks, opossums could be used as bioindicators in Brazilian zoonotic areas with spotted fever. Considering the prevalence and also abundance of ticks, host attractiveness, proliferation and susceptibly for $R$. rickettsi infection, capybaras and opossums are the main amplifying hosts for this microorganism at the ESALQ/Campus, while horses, black vultures and stray cats act as secondary hosts.
\end{abstract}

KEY WORDS: Amblyomma spp., mammals, birds, reservoir hosts, bioindicators

\section{RESUMO}

Foram avaliadas 7 espécies da mastofauna e 36 da avifauna quanto à prevalência e intensidade de infestação por carrapatos na ESALQ/USP, no Município de Piracicaba, SP. Analisa-

*Parte da Tese de Doutorado do primeiro autor, Programa de Pós-Graduação em Ciências Florestais da Escola Superior de Agricultura Luiz de Queiroz (ESALQ/USP), auxílio CNPq (processo No. 141318/ 2005-8, and Academic career scholarship to D.M.B.B.).

${ }^{1}$ Escola Superior de Agricultura Luiz de Queiroz, Av. Pádua Dias 11, CP 09, Piracicaba, SP 13418-900, Brasil. E.mail: c aa_perez@hotmail.com

${ }^{2}$ Biométrica, Avaliações Biológicas e Manejo Ambiental, Piracicaba, SP.

${ }^{3}$ Departamento de Engenharia Ambiental, Escola de Engenharia de Piracicaba, SP.

${ }^{4}$ Laboratório de Parasitologia, Instituto Butantan, Av. Vital Brasil 1500, São Paulo, SP 05503-900, Brasil ram-se 52 indivíduos da mastofauna e 158 da avifauna, parasitados por 12418 carrapatos. Os exemplares adultos $(\mathrm{N}=$ 7343) foram encontrados em parasitismo nas capivaras enquanto que os imaturos foram, na maioria, coletados de pequenos mamíferos e aves. Os principais hospedeiros para as formas imaturas, em ordem decrescente, foram gambás $(69,1 \%)$, capivaras $(24,4 \%)$ e urubus (3,7\%). Entre a avifauna, o urubu apresentou o maior número de carrapatos com 69,9\%, seguido por indivíduos das famílias Thamnophilidae e Turdidae. Os carrapatos adultos encontrados em capivaras foram A. cajennense (80,8\%) e A. dubitatum (19,2\%). Ambas as espécies foram também coletadas em gambás, correspondendo a $72,4 \%$ e $27,6 \%$, respectivamente. Pela facilidade de captura e atratividade de Amblyomma spp. o gambá 
pode ser usado como bioindicador de infestação em locais endêmicos para febre maculosa. Considerando os índices de parasitismo e prevalência bem como de abundância de carrapatos, susceptibilidade dos hospedeiros, proliferação e susceptibilidade para infecção por $R$. rickettsi, capivaras e gambás são potenciais hospedeiros amplificadores desse microrganismo no Campus da ESALQ, enquanto eqüídeos, urubus e gatos atuam como hospedeiros secundários.

PALAVRAS-CHAVE: Amblyomma spp., mamíferos, aves, reservatórios, bioindicadores.

\section{INTRODUÇÃO}

A transmissão de Rickettsia rickettsii na maior parte das áreas endêmicas no Estado de São Paulo continua sendo atribuída principalmente ao carrapato Amblyomma cajennense (Fabricius, 1787). Este carrapato possui baixa especificidade parasitária, principalmente nos estágios de larva e ninfa, parasitando indistintamente diferentes classes animais incluindo humanos. A segunda espécie responsável pela transmissão do agente etiológico em determinados locais é A. aureolatum (Pallas, 1772) (PINTER et al., 2004). No entanto algumas espécies de carrapato, como A. dubitatum Neumann, 1899, desempenham uma importância secundária na epidemiologia da doença, porém, são importantes na manutenção e amplificação da bactéria $R$. rickettsii na natureza (SUPERINTENDÊNCIA DE CONTROLE DE ENDEMIAS, 2004; SANGIONI et al., 2005). Segundo Labruna e Machado (2006), A. dubitatum pode também apresentar infecção por Rickettsia parkeri que possui patogenicidade branda, ou ainda por outra espécie de Rickettsia de patogenicidade desconhecida (LABRUNA et al., 2004; GUGLIELMONE et al., 2006).

Os carrapatos são artrópodes desprovidos de capacidade própria de locomoção à grandes distâncias, adultos de algumas espécies de Ixodidae podem se dispersar entre 2 a $10 \mathrm{~m}$ do lugar em que são soltos (WILSON et al., 1972; FALCO; FISH,1991). Já a dispersão de formas imaturas se limita a distâncias de poucos centímetros a partir do lugar onde emergiram as larvas ou ninfas (DANIELS; FISH, 1990).

Porém carrapatos da família Ixodidae possuem altas taxas de reprodução e podem ser transportados por longas distâncias através de seus hospedeiros. Desta forma, a fauna silvestre participa de forma decisiva nessa atividade, promovendo a disseminação e adaptação dos carrapatos a diversos tipos de ambientes. Mesmo que, devido às suas exigências ambientais, os carrapatos sobrevivam em pequenas parcelas, ainda assim, elas serão suficientes para dar continuidade ao ciclo biológico nos novos nichos (ROHR, 1909; MOREIRA; MAGALHAES, 1935; CLOUDSLEY-THOMPSON, 1980).

Nesse contexto, a capivara que é um animal silvestre amparado pela Lei 5.197/67 de proteção à fauna tem contribuído enormemente para garantir o sucesso do crescimento populacional de algumas espécies de carrapatos (BRASIL, 1967; PINTO, 2003), especialmente aquelas associadas à Febre Maculosa.
Para que uma população de $A$. cajennense se estabeleça no ambiente é necessário a presença de dois tipos de hospedeiros, os primários: capivara, eqüinos e anta- e os secundários, dezenas de espécies da mastofauna e avifauna. Os eqüinos, embora considerados hospedeiros primários de A. cajennense, e compartilharem eventualmente o mesmo ambiente com capivaras, quando pulverizados semanalmente com carrapaticidas entre os meses de abril e outubro, podem reduzir o potencial na disseminação de carrapatos no local (LABRUNA et al., 2002; LEITE et al., 2006). Infestações experimentais de $A$. dubitatum em varias espécies de aves e mamíferos mostraram que a capivara foi o melhor hospedeiro para formas imaturas. Fêmeas adultas desse carrapato, ao parasitarem capivaras, mostraram elevada capacidade de ingurgitamento e de peso das massas de ovos, indicando que esse roedor é um hospedeiro primário para $A$. dubitatum (LABRUNA et al., 2004).

Várias espécies de carrapatos podem coabitar no mesmo hospedeiro. Cães que entram na mata ou que compartilham seu ambiente com a fauna silvestre (SZABÓ et al., 2001), podem apresentar infestações mistas, por exemplo, A. cajennense, Amblyomma ovale Koch, 1844 e Rhiphicephalus sanguineus (Latreille, 1806). Da mesma forma, pequenos mamíferos silvestres que vivem próximos aos domicílios também podem apresentar dupla infestação. Barros-Battesti et al. (2000) verificaram a presença de A. cajennense e Ixodes loricatus Neumann, 1899 no gambá, assim como Arzua et al. (2003) observaram dupla infestação em aves passeriformes por A. aureolatum e I. auritulus Neumann, 1904.

Para que uma espécie de vertebrado seja considerada um bom hospedeiro amplificador de $R$. rickettsii na natureza, ela deve preencher alguns atributos: ser abundante na área endêmica para febre maculosa; ser um bom hospedeiro do carrapato vetor em condições naturais; ser susceptível à infecção por $R$. rickettsii, manter a bactéria circulante em níveis plasmáticos suficientes para infectar carrapatos que se alimentam nele e ter uma alta taxa de renovação populacional (BURGDORFER, 1988; LABRUNA, 2006).

Considerando as altas populações de carrapatos no Campus Luiz de Queiroz da Escola Superior de Agricultura (ESALQ/ USP), localizado no município de Piracicaba- SP (ponto central a $22^{\circ} 42^{\prime}$ Sul e $47^{\circ} 38^{\prime}$ ' Oeste) e os casos fatais de Febre Maculosa (FM) nos últimos anos, o presente estudo teve por objetivos avaliar o índice de infestação de carrapatos em parasitismo na avifauna e mastofauna; identificar os hospedeiros primários e secundários e classificar os principais hospedeiros potenciais quanto à importância qualitativa de efeito amplificador para manutenção da $R$. rickettsii.

\section{MATERIAL E MÉTODOS}

\section{Captura de mamíferos e carrapatos}

Três capivaras foram capturadas em bretes utilizando espigas de milho como ceva durante o mês de janeiro de 2006, e foram sacrificadas para realização da contagem dos carra- 
patos presos à pele. Os couros foram então removidos e colocados em sacos plásticos, os quais foram mantidos durante três dias a $-10{ }^{\circ} \mathrm{C}$. Registrou-se o número de carrapatos encontrados no lado esquerdo da superfície corpórea, sendo este número multiplicado por dois para estimar o total infestante por animal, seguindo as orientações de Oliveira (1998).

Pequenos mamíferos foram capturados com armadilhas de tamanho pequeno (11 x 11 x $20 \mathrm{~cm}$ ) e médio ( 25 x 25 x 40 $\mathrm{cm})$ com suporte para iscas suspensas. As armadilhas foram distribuídas em três áreas escolhidas aleatoriamente, todas freqüentadas por capivaras, quinze dias antes do início das capturas no campo. As maiores foram colocadas em transectos no solo, a cada $20 \mathrm{~m}$, sendo as menores dispostas até $2 \mathrm{~m}$ de altura, presas às árvores ou aos troncos caídos. Foram utilizadas iscas de fubá, óleo de fígado de bacalhau, paçoca de amendoim, óleo de soja e banana.

As capturas dos pequenos mamíferos foram efetuadas duas vezes por mês, durante sete dias consecutivos, no período de abril a junho de 2006, nos três locais selecionados, sendo vistoriadas diariamente, totalizando um esforço de captura de 2.240 armadilhas/dia.

Os mamíferos parasitados por carrapatos foram mantidos por 7 dias em caixas plásticas de poliestireno, forradas com maravalha (substrato), para permitir o desprendimento natural dos carrapatos após o ingurgitamento. Diariamente foi realizada a higienização das caixas com troca do substrato, reposição de alimento e de água. Os carrapatos imaturos que se desprenderam naturalmente foram colhidos e acondicionados em tubos plásticos tipo "Eppendorf”, e estes foram tampados com chumaço de algodão umedecido em água. A seguir foram mantidos em incubadora BOD a $27 \pm 2{ }^{\circ} \mathrm{C}$ com umidade relativa de $85 \%$ e 12/12 horas de fotoperíodo, até o exemplar sofrer muda para o estágio seguinte.

Todas as capturas de animais silvestres foram efetuadas mediante autorização do Ibama, No 249/06 GEREX-SP/Fauna/ LIC.

Também foi avaliado o estado de ectoparasitismo de gatos errantes que vivem nas imediações do Campus Luiz de Queiroz. Tais observações foram realizadas no mês de junho de 2006 e seguiram a mesma metodologia empregada com os animais silvestres.

Seis eqüinos de dois grupos foram observados: três usados em atividades de lida diária e três empregados no programa de equoterapia. Nestes animais registrou-se o número de carrapatos encontrados no lado esquerdo do corpo, e o número encontrado foi multiplicado por dois para estimar o total infestante por animal.

\section{Captura de aves e carrapatos}

Os urubus (Coragyps atratus Bechstein) foram igualmente investigados, por permanecerem constantemente próximos aos grupos de capivaras, das quais aproveitam restos de placenta e eventualmente de filhotes. A captura foi realizada por meio de uma estrutura quadrada de madeira de 4,5 x 4,5 m telada com nylon. Na base dessa estrutura foi acondicionada uma carcaça de bovino para servir de atrativo. A estrutura foi erguida e amarrada com uma corda que foi solta no momento em que um grupo de urubus se encontrava em plena atividade de forrageio. Tal captura foi realizada em agosto de 2006, época de pico populacional de ninfas de $A$. cajennense (LABRUNA et al., 2004).

Foi avaliada a infestação em aves silvestres por meio de capturas em duas épocas diferentes. A primeira correspondeu ao pico sazonal de adultos e a segunda ao de larvas de $A$. cajennense, nos meses de janeiro e maio de 2007, respectivamente (LABRUNA et al., 2004). Para cada época foram usadas três estações de coleta em locais diferentes, escolhidas aleatoriamente.

As aves foram capturadas com "redes de neblina”, sendo utilizadas 15 unidades medindo $12 \mathrm{~m} \mathrm{X} \mathrm{2,5m} \mathrm{com} \mathrm{malha} \mathrm{de}$ $36 \mathrm{~mm}$, cada. As redes foram instaladas no sub-bosque, permanecendo abertas por um período de cinco horas a partir do nascer do sol, sendo amostrada uma estação por dia, durante três dias consecutivos com esforço de captura total de 1.350 horas-rede, nas duas épocas de coleta.

As aves capturadas foram identificadas utilizando os guias de campo de Meyer de Schauensee (1983), Narosky e Yzurieta (1993), Sick (1997) e Souza (1998). Após serem examinadas quanto à presença de ectoparasitos e marcadas com anilhas metálicas, elas foram liberadas.

De acordo com o uso posterior dos carrapatos, ao serem retirados das aves, eles eram colocados em recipientes de vidro rotulados e fixados em solução de álcool $80 \%$, ou congelados a $-20{ }^{\circ} \mathrm{C}$, ou ainda acondicionados em tubos plásticos tampados com chumaço de algodão umedecido com água. Neste último caso, eles foram mantidos vivos em incubadora BOD a $27 \pm 2{ }^{\circ} \mathrm{C}$ e umidade relativa de $85 \%$, até se processarem as mudas para os estágios seguintes.

Os carrapatos encontrados nos hospedeiros foram analisados de acordo com estágio de desenvolvimento, prevalência, amplitude - de parasitismo, e abundância de ectoparasitos, seguindo o modelo proposto por Margolis et al. (1982).

\section{RESULTADOS}

No período de março de 2006 a maio de 2007 foram investigados 210 indivíduos, sendo 52 pertencentes a 7 espécies da mastofauna e 158 indivíduos incluídos em 36 espécies pertencentes a 16 famílias da avifauna. Nesses hospedeiros foram coletados 12.418 carrapatos adultos e imaturos. Aqueles que chegaram ao estágio adulto foram identificados como A. cajennense, $A$. dubitatum e $A$. nodosum Neumann 1899. Os carrapatos imaturos que morreram antes da muda foram mantidos como Amblyomma sp. Os carrapatos adultos corresponderam a 7.343 indivíduos, sendo a maioria coletada nas capivaras, enquanto que as formas imaturas totalizaram 5.075 exemplares, os quais foram obtidos principalmente em pequenos mamíferos e aves.

O número de carrapatos encontrados em cada espécie de hospedeiro da mastofauna, e sua respectiva prevalência e variação de parasitismo por espécie, são mostrados na Tabela 1. 
Tabela 1. Prevalência, abundância e variação de infestação de Amblyomma spp. em hospedeiros da mastofauna no Campus Luiz de Queiroz. Piracicaba, janeiro de 2006 a maio de 2007.

\begin{tabular}{|c|c|c|c|c|c|c|}
\hline $\begin{array}{c}\text { Grupo /Ordem } \\
\text { Espécie (nome comum) }\end{array}$ & $\begin{array}{l}\text { Indivíduos } \\
\text { examinados }\end{array}$ & $\begin{array}{c}\text { Prevalência } \\
\mathrm{n}(\%)^{1}\end{array}$ & $\begin{array}{l}\text { Número de } \\
\text { Imaturos }\end{array}$ & $\begin{array}{c}\text { carrapatos } \\
\text { Adultos }\end{array}$ & $\begin{array}{l}\text { Abundância } \\
\text { Média (SD) }\end{array}$ & Variação \\
\hline $\begin{array}{l}\text { Hydrochaeris hydrochaeris } \\
\text { Rodentia, Hydrochaeridae, } \\
\text { Capivara }\end{array}$ & 3 & $3(100)$ & 1.239 & 7.343 & 2.860 & $211-3.158$ \\
\hline $\begin{array}{l}\text { Rattus rattus } \\
\text { Rodentia, Muridae, } \\
\text { Rato de telhado }\end{array}$ & 5 & $3(60)$ & 29 & 0 & 5,8 & $0-9$ \\
\hline $\begin{array}{l}\text { Rattus norvergicus } \\
\text { Rodentia, Muridae } \\
\text { Ratazana }\end{array}$ & 2 & 0 & 0 & 0 & 0 & 0 \\
\hline $\begin{array}{l}\text { Didelphis albiventris } \\
\text { Marsupialia, Didelphidae, } \\
\text { Gambá }\end{array}$ & 26 & $21(80,8)$ & 3.510 & 0 & 135 & $0-916$ \\
\hline $\begin{array}{l}\text { Nasua nasua } \\
\text { Procyonidae, Carnivora } \\
\text { Coati }\end{array}$ & 3 & 0 & 0 & 0 & 0 & 0 \\
\hline $\begin{array}{l}\text { Felis catus } \\
\text { Carnivora, Felidae } \\
\text { Gato }\end{array}$ & 7 & $6(85,7)$ & 28 & 0 & 4 & $0-11$ \\
\hline $\begin{array}{l}\text { Equus caballus } \\
\text { Perissiodactyla, Equidae } \\
\text { Cavalo (Lida) }\end{array}$ & 3 & $1(33,3)$ & $6^{2}$ & $0^{3}$ & 1 & $0-6$ \\
\hline $\begin{array}{l}\text { Equus caballus } \\
\text { Equidae, Perissiodactyla } \\
\text { Cavalo (Equoterapia) }\end{array}$ & 3 & 0 & 0 & 0 & & \\
\hline
\end{tabular}

${ }^{1} \mathrm{n}=$ número de indivíduos infestados (porcentagem infestada/espécie).

${ }^{2}$ Encontrados 74 imaturos e ${ }^{3} 16$ adultos de Dermacentor nitens (Neumann, 1897) ${ }^{4}$ SD (Desvio padrão).

Nos três exemplares de capivaras avaliados foi encontrado um total de 7.343 adultos e 1.239 formas imaturas com 100\% de prevalência e abundância média de 2860,7士389,6 carrapatos (Tabela 1). De uma amostra ( $\mathrm{n}=500)$ de carrapatos adultos encontrados nesses hospedeiros, noventa e seis carrapatos, correspondendo a 19,2\%, foram identificados como $A$. dubitatum, sendo a maioria, A. cajennense ( $80,8 \%)$. Os carrapatos adultos se encontravam concentrados principalmente na nuca, até a parte mediana do dorso e na região ventral. Ninfas e larvas foram observadas distribuídas por todo o corpo.

Em 26 exemplares de gambá avaliados no pico sazonal de larvas de A. cajennense, foram encontradas 3.510 formas imaturas de Amblyomma spp., com 80,8\% de prevalência e abundância média de $135 \pm 47,3$ carrapatos. Nenhum adulto foi registrado parasitando esses animais (Tabela 1). Foi observado que a fixação dos carrapatos em gambás se concentra principalmente atrás das orelhas. De um lote de formas imaturas $(\mathrm{n}=263)$ parasitando gambás (transformadas em adultos em condições de laboratório), 72,4\% correspondeu a A. cajennense e $27,6 \%$ a $A$. dubitatum.

Nos eqüinos de lida foram encontradas 6 ninfas do gênero Amblyomma . Nos três cavalos usados no programa de equoterapia, nenhum exemplar de carrapato foi encontrado.

As observações realizadas na avifauna, sua respectiva prevalência e variação de parasitismo por espécie são mostradas na Tabela 2.
Nas avaliações efetuadas em aves silvestres durante a fase sazonal de adultos de $A$. cajennense não foram encontrados espécimes parasitando aves. Já no pico sazonal de larvas de A. cajennense, observou-se parasitismo em urubus e em pequenas aves no período de março a julho de 2006 e de 2007, respectivamente. Do total de carrapatos coletados neste trabalho, 2,1\% ( $\mathrm{n}=266)$ foram coletados de aves e corresponderam a formas imaturas de Amblyomma, os quais foram encontrados fixados na região perioftálmica (Tabela 2).

Uma ninfa de $A$. nodosum foi encontrada parasitando Haplospiza unicolor (cigarra-bambu) (Emberezidae) ave granívora, de borda de mata, de hábito médio inferior. Esta espécie de carrapato, no estágio adulto, parasita principalmente tamanduás (Xenarthra), embora larvas e ninfas já tenham sido registradas em aves (ONOFRIO et al., 2006; MARTINS et al., 2004; BITENCOURTH et al., 2007; SERRA-FREIRE et al., 1993). As espécies da família Picidae de hábitos geralmente associados ao dossel, como o pica-pau-rei, não apresentaram infestação por carrapatos.

Ainda que no Brasil o papel de espécies animais envolvidas como amplificadoras na transmissão efetiva de $R$. rickettsii, tenha sido largamente ignorado, a Tabela 3 mostra uma lista de hospedeiros que habitam no Campus Luiz de Queiroz, os quais reúnem alguns atributos, segundo Labruna (2006), para serem considerados potenciais amplificadores da bactéria. 
Tabela 2. Prevalência, abundância e variação de infestação de Amblyomma sp. em hospedeiros da Avifauna no Campus Luiz de Queiroz. Piracicaba, janeiro de 2006 a maio de 2007.

\begin{tabular}{|c|c|c|c|c|c|c|}
\hline \multirow{2}{*}{$\begin{array}{c}\text { Grupo /Familia } \\
\text { Espécie (nome comum) }\end{array}$} & \multirow{2}{*}{$\begin{array}{l}\text { Indivíduos } \\
\text { examinados }\end{array}$} & \multirow{2}{*}{$\begin{array}{l}\text { Prevalência } \\
\mathrm{n}(\%)^{1}\end{array}$} & \multicolumn{2}{|c|}{ Número de carrapatos } & \multirow{2}{*}{$\begin{array}{l}\text { Abundância } \\
\text { Média(SD) }^{2}\end{array}$} & \multirow[t]{2}{*}{ Variação } \\
\hline & & & Imaturos & Adultos & & \\
\hline \multicolumn{7}{|l|}{ Familia Cathartidae } \\
\hline Coragyps atratus (Urubu) & 12 & $11(91,7)$ & 186 & 0 & 15,5 & $0-46$ \\
\hline \multicolumn{7}{|l|}{ Familia Rallidae } \\
\hline Aramides saracura (Saracura) & 1 & $1(100)$ & 8 & 0 & 8 & 8 \\
\hline \multicolumn{7}{|l|}{ Familia Collumbidae } \\
\hline Leptotila verreauxi (Juriti) & 10 & 0 & 0 & 0 & 0 & 0 \\
\hline Leptotila rufaxilla (Juriti) & 3 & 0 & 0 & 0 & 0 & 0 \\
\hline \multicolumn{7}{|l|}{ Familia Picidae } \\
\hline Veniliornis spilogaster (Pica-pau-carijó) & 2 & 0 & 0 & 0 & 0 & 0 \\
\hline Veniliornis passerinus (Pica-pau-carijó) & 1 & 0 & 0 & 0 & 0 & 0 \\
\hline Picumnus cirratus (Pica-pau-anão) & 9 & $4(44,4)$ & 7 & 0 & 0,8 & $0-4$ \\
\hline Campephilus robustus (Pica-pau-rei) & 1 & 0 & 0 & 0 & 0 & 0 \\
\hline \multicolumn{7}{|l|}{ Familia Thamnophilidae } \\
\hline Taraba major (Choró-boi) & 2 & 0 & 0 & 0 & 0 & 0 \\
\hline Thamnophilus caerulescens (Choca-da-mata) & 15 & $4(26,7)$ & 29 & 0 & 1,93 & $0-14$ \\
\hline Thamnophilus doliatus (Chóca-barrada) & 5 & $1(20)$ & 3 & 0 & 0,6 & $0-3$ \\
\hline \multicolumn{7}{|l|}{ Família Thraupidae } \\
\hline Rhamphocelus carbo (Pipira-vermelha) & 4 & $2(50)$ & 3 & 0 & 12,5 & $0-2$ \\
\hline Thlypopsis sordida (Saíra) & 7 & $2(28,6)$ & 7 & 0 & 1 & $0-5$ \\
\hline Thraupis sayaca (Sanhaço) & 9 & 0 & 0 & 0 & 0 & 0 \\
\hline Thachyphonus coronatus (Tié-preto) & 9 & $1(11,1)$ & 3 & 0 & 0,3 & $0-3$ \\
\hline \multicolumn{7}{|l|}{ Família Parulidae } \\
\hline Basileuterus flaveolus (Pula-pula) & 8 & $2(25)$ & 3 & 0 & 0,37 & $0-2$ \\
\hline Basileuterus culicivorus (Pula-pula) & 9 & 0 & 0 & 0 & 0 & 0 \\
\hline \multicolumn{7}{|l|}{ Família Tyraniidae } \\
\hline $\begin{array}{l}\text { Tolmomyias sulphurescens } \\
\text { (Bico-chato-de-orelha-preta) }\end{array}$ & 2 & 0 & 0 & 0 & 0 & 0 \\
\hline Pitangus sulphuratus (Bem-te-vi) & 5 & 0 & 0 & 0 & 0 & 0 \\
\hline Lathrotriccus euleri (Enferrujado) & 2 & 0 & 0 & 0 & 0 & 0 \\
\hline Platyrhinchus mystaceus(Patinho) & 2 & $1(50)$ & 2 & 0 & 1 & $0-2$ \\
\hline \multicolumn{7}{|l|}{ Família Muscicapidae } \\
\hline Turdus rufiventris (Sabiá-laranjeira) & 1 & 0 & 0 & 0 & 0 & 0 \\
\hline Turdus leucomelas (Sabiá-barranco) & 17 & $1(5,9)$ & 10 & 0 & 0,6 & $0-10$ \\
\hline Turdus amaurochalinus (Sabiá-póca) & 1 & 0 & 0 & 0 & 0 & 0 \\
\hline \multicolumn{7}{|l|}{ Familia Corvidae } \\
\hline Cyanocorax cristatellus (Gralha-do-campo) & 1 & $1(100)$ & 4 & 0 & 4 & 4 \\
\hline \multicolumn{7}{|l|}{ Família Conopophagidae } \\
\hline Conopophaga lineata (Chupa-dente) & 8 & 0 & 0 & 0 & 0 & 0 \\
\hline \multicolumn{7}{|l|}{ Família Furnariidae } \\
\hline Synalaxis sp. (Pichororé) & 1 & 0 & 0 & 0 & 0 & 0 \\
\hline Automolus leucophtalmus (Barranqueiro) & 2 & 0 & 0 & 0 & 0 & 0 \\
\hline \multicolumn{7}{|l|}{ Família Emberizidae } \\
\hline Sporophila sp. (Cigarra) & 1 & 0 & 0 & 0 & 0 & 0 \\
\hline Coereba flaveola (Cambacica) & 1 & 0 & 0 & 0 & 0 & 0 \\
\hline Haplospiza unicolor* (Cigarra-bambu) & 1 & $1(100)$ & 1 & 0 & 1 & 1 \\
\hline Zonotrichia capensis (Tico-tico) & 1 & 0 & 0 & 0 & 0 & 0 \\
\hline Família Troglodytidae & & & & & & \\
\hline Troglodytes musculus (Corruíra) & 2 & 0 & 0 & 0 & 0 & 0 \\
\hline Família Fringillidae & & & & & & \\
\hline Euphonia violacea (Gaturamo-verdadeiro) & 2 & 0 & 0 & 0 & 0 & 0 \\
\hline Familia Alcedinidae & & & & & & \\
\hline Chloroceryle americana (Martim-pescador) & 1 & 0 & 0 & 0 & 0 & 0 \\
\hline
\end{tabular}
vio padrão)

Tabela 3. Espécies potenciais que poderiam estar envolvidas na manutenção do ciclo vital da $R$. ricketsii na natureza no Campus Luiz de Queiroz*.

\begin{tabular}{|c|c|c|c|c|c|}
\hline Item & Capivara & Gambá & Cavalo & Urubu & Gato \\
\hline $\begin{array}{l}\text { 1. Ser abundante na área } \\
\text { endêmica }\end{array}$ & Sim & Sim & Sim & Sim & Sim \\
\hline $\begin{array}{l}\text { 2. Ser bom hospedeiro do } \\
\text { carrapato vetor }\end{array}$ & Sim & Sim & Sim & Sim & Não \\
\hline $\begin{array}{l}\text { 3. Ser susceptível a infecção } \\
\text { por R. rickettsii }\end{array}$ & Sim & Sim & Sim & ? & ? \\
\hline 4. Manter a bactéria circulante & ? & $?$ & $?$ & $?$ & $?$ \\
\hline 5. Ter alta taxa de renovação & Sim & Sim & Não & Não & Sim \\
\hline
\end{tabular}

*Adaptado de Labruna (2006)

\section{DISCUSSÃO}

Apesar da pouca diversidade de mastofauna silvestre (GHELER-COSTA, 2002), o Campus Luiz de Queiroz apresenta ambiente físico e biológico propício para o desenvolvimento de carrapatos e muitas capivaras. Em três exemplares de capivaras capturadas na natureza, os 8.582 exemplares de carrapatos, superam os valores já encontrados por Souza et al. (2004) em Campinas e por Ferreira (2006) em Franca, SP.

As capivaras e os gambás concentraram a quase totalidade de carrapatos da mastofauna avaliada, sendo as capivaras parasitadas em sua maioria por adultos A. cajennense, e so- 
mente uma pequena porcentagem correspondeu a $A$. dubitatum. Souza et al. (2004) verificaram maior ocorrência de A. dubitatum (59\%) parasitando capivaras quando comparada com a infestação de $A$. cajennense (41\%) para o município de Campinas. Tal diferença encontrada no presente estudo pode ter sido influenciada pela época da captura das capivaras que ocorreu no mês de janeiro, durante o maior pico sazonal de adultos de A. cajennense (LABRUNA et al., 2004). Souza et al. (2006) fizeram uma avaliação na vegetação do Campus da Esalq e verificaram maior número de A. cajennense em relação a A. dubitatum. No entanto, os autores não especificaram quais proporções foram encontradas para essas espécies, quando em vida livre.

Os gambás mostraram-se infestados apenas pelos estágios imaturos de A. cajennense e de A. dubitatum, estes em menor porcentagem, estando de acordo com os achados de Horta (2006b). Dentre os mamíferos capturados na natureza, eles foram os mais parasitados depois das capivaras. Por serem de porte pequeno e terem hábitos crepusculares e noturnos, esses animais percorrem facilmente as áreas do Campus, aumentando as possibilidades de dispersão ou recolonização de carrapatos em lugares onde as capivaras não têm acesso. Situação semelhante foi também observada por Pinter et al. (2006) no Guarujá, SP, para a espécie A. fuscum Neumann, 1907. Os autores constataram que a presença deste carrapato nos domicílios, devia-se aos gambás que atuavam como transportadores da espécie das áreas de mata para as residências. Desta forma os gambás podem ser utilizados como bioindicadores do estado de infestação de Amblyomma spp.

É de se considerar que pela alta capacidade de abrigar grandes populações de Amblyomma spp, as capivaras e gambás representem grande potencial como dispersores e amplificadores para manter o ciclo vital da $R$. rickettsii na natureza (LABRUNA, 2006; HORTA, 2006a), principalmente pelo fato de o gambá abrigar formas imaturas e a capivara adultos e secundariamente ninfas.

Quanto aos animais domésticos, nos gatos capturados, a maioria se encontrava parasitada por ninfas de Amblyomma spp. Segundo Campos (2004), os cães e gatos errantes que predam animais silvestres no Campus, consomem até 25,4 e 2,9 kg/indivíduo/ano respectivamente. Considerando que cães são susceptíveis à infecção por $R$. rickettsii (MOREIRA; MAGALHÃES, 1935) por meio da ingestão de carne de cobaias infectadas com a bactéria, isto é, sem a participação do vetor, medidas de prevenção e monitoramento se fazem necessários para verificar a possibilidade de esses animais errantes representarem risco como potenciais reservatórios de doenças. Tanto que, na Venezuela foram encontrados gatos e cães freqüentemente parasitados por $A$. cajennense em áreas urbanas e periurbanas, aumentando o risco de infestação em humanos (MOISSANT et al., 2002).

A avaliação realizada em eqüinos confirma o controle satisfatório de carrapatos de vida parasitária realizado rotineiramente entre março/abril e outubro/novembro de acordo com o nível de infestação observado nos animais.
O parasitismo de Amblyomma spp. em urubus em área endêmica para Febre Maculosa no Brasil está sendo registrado aqui pela primeira vez. O urubu seguido do gambá e da capivara foi o hospedeiro mais intensamente parasitado pelas formas imaturas de Amblyomma spp. abrigando quase que a totalidade de carrapatos coletados em aves. Serafini et al. (2003) estudando o ácaro Ornithonyssus sylviarum Canestrini e Fanzago (Macronyssidae) em urubus, destacaram o importante papel desta espécie na comunidade de aves de ambientes silvestres e sua interrelação com aves urbanas. Segundo os autores, os urubus podem agir como vetores em ambas as direções, afetando a dinâmica e a saúde dos ecossistemas silvestres. As aves pertencentes famílias Thamnophilidae, Thraupidae e Turdidae também se mostraram parasitadas, embora com índices mais baixos de infestação. O fato de voarem próximo ao chão para obtenção do alimento resulta em um maior contato com carrapatos de vida livre, aumentando o risco de parasitismo.

Das espécies potenciais para manutenção do ciclo vital da $R$. rickettsii, pode-se inferir que a capivara e o gambá são as eleitas no Campus Luiz de Queiroz , reunindo a maior parte das condições apontadas por Labruna (2006), uma vez que são altamente suscetíveis ao parasitismo por Amblyomma spp. Segundo Ojatsi (1973) e Emmons e Feer (1997), além de prolíferos, esses hospedeiros apresentam alta taxa de renovação populacional. Dessa forma, capivaras e gambás possibilitam constantemente a introdução regular de animais susceptíveis na população.

\section{REFERÊNCIAS BIBLIOGRÁFICAS}

ARAGÃO, H. Ixodidas brasileiros e de alguns paizes limitrophes. Memórias do Instituto Oswaldo Cruz, v. 31, n. 4, p. 759-843, 1936.

ARZUA, M.; SILVA, M.A.N.; FAMADAS, K.M.; BEATI, L.; BARROS-BATTESTI, D.M. Amblyomma aureolatum and Ixodes auritulus (Acari: Ixodidae) on birds in southern Brazil, with notes on their ecology. Experimental and Applied Acarology, v. 31, n. 3-4, p. 283-296, 2003.

BARROS-BATTESTI, D.M.; YOSHINARI, N.H.; BONOLDI, V.L.N.; GOMES, A.C. Parasitism by Ixodes didelphidis and I. loricatus (Acari: Ixodidae) on Small Wild Mammals from an Atlantic Forest in the State of São Paulo, Brazil. Journal of Medical Entomology, v. 37, n. 6, p. 820-827, 2000.

BITENCOURTH, K.; TEIXEIRA, R.H.F.; AMORIN, M.; GAZETA, G.S.; SERRA-FREIRE, N.M. Análise do número de cerdas internas do órgão de Haller de larvas de Amblyomma nodosum Neumann (Acari: Ixodidae). Revista Brasileira de Entomologia, v. 51, n. 1, p. 58-61, 2007.

BRASIL. Lei no 5.197 de 03 de janeiro de 1967. Dispõe sobre a proteção à fauna e dá outras providências. Diário Oficial [da] Republica Federativa do Brasil, Poder Executivo, Brasília, DF, 05 jan. 1967. Seção 1, p. 177.

BURGDORFER, W. Ecological and epidemiological 
considerations of Rocky Mountain spotted fever and scrub typhus. In: WALKER, D.H. Biology of Rickettsial Diseases, Boca Raton: CRC, 1988. v. 1, p. 33-50.

CAMPOS, C.B. Impacto de cães (Canis familiaris) e gatos (Felis catus) errantes sobre a fauna silvestre em ambiente peri-urbano. 2004. 55f. Dissertação (Mestrado) - Escola Superior de Agricultura “Luiz de Queiroz”, USP, Piracicaba, 2004

CLOUDSLEY-THOMPSON, J.I. Microecologia. São Paulo: EPU, 1980. 58p.

DANIELS, T.J.; FISH, D. Spatial distribution and dispersal of unfed larval Ixodes dammini (Acari: Ixodidae) in southern New York. Environmental Entomology, v. 19, n. 4, p. 1029-1033, 1990.

EMMONS, L.H.; FEER, F. Neotropical rainforest mammals a field guide. 2nd ed. London: The University of Chicago Press, 1997. 307 p.

FALCO, R.C.; FISH, D. Horizontal movement of adult Ixodes dammini (Acari: Ixodidae) attracted to $\mathrm{CO}_{2}$ baited traps. Journal of Medical Entomology, v. 28, n. 5, p. 726-729, 1991.

FERREIRA, A.A. Carrapatos em roedores da região de Franca-SP: Avaliação preliminar do potencial de transmissão de doenças infecciosas para a população humana. 2006. 49 f. Dissertação (Mestrado) - Universidade de Franca, Franca, 2006.

GHELER-COSTA, C. Mamíferos não-voadores do Campus “Luiz de Queiroz”, da Universidade de São Paulo, em Piracicaba, Estado de São Paulo. 2002. 72 f. Dissertação (Mestrado) - Escola Superior de Agricultura "Luiz de Queiroz”, USP, Piracicaba, 2002.

GUGLIELMONE, A.A.; BEATI, L.; BARROS-BATTESTI, D.M.; LABRUNA, M.B.; NAVA, S.; VENZAL, J.M.; MANGOLD, A.J.; SZABO, M.P.J.; MARTINS, J.R.; GONZALEZ-ACUNA, D.; ESTRADA-PENA, A. Ticks (Ixodidae) on humans in South America. Experimental and Applied Acarology, v. 40, n. 2, p. 83-100, 2006.

HORTA, M.C. Estudo epidemiológico de Rickettsia felis em áreas endêmicas e não-endêmicas para febre maculosa no Estado de São Paulo. 2006. 106 f. Tese (Doutorado) - Faculdade de Medicina Veterinária e Zootecnia, USP, São Paulo. 2006a.

HORTA, M.C. Gambás e febre maculosa. In: CONGRESSO BRASILEIRO DE PARASITOLOGIA VETERINÁRIA $14 \mathrm{e}$ SIMPÓSIO LATINO-AMERICANODE RICKETTSIOSES, 2, 2006, Ribeirão Preto. Resumos... Jaboticabal: CBPV: 2006b. p. 156.

LABRUNA, M.B.; KASAI, N.; FERREIRA, F.; FACCINI, J.L.H.; GENNARI, S.M. Seasonal dynamics of ticks (Acari: Ixodidae) on horses in the state of São Paulo, Brasil. Veterinary Parasitology, v. 105, n. 1, p. 65-77, 2002.

LABRUNA, M.B.; LEITE, R.C.; GOBESSO, A.A.O.; GENARI, S.M.; KASAI, N. Strategic control of the tick Amblyomma cajennense on horses. Ciencia Rural, v. 34, n. 1, p. 195-200, 2004.
LABRUNA, M.B. Epidemiologia da Febre Maculosa no Brasil e nas Américas. In: SIMPÓSIO BRASILEIRO DE ACAROLOGIA. 2006, Viçosa. Anais... Viçosa: UFV, 2006. p. 63.

LABRUNA, M.B.; MACHADO, R.Z. Agentes transmitidos por carrapatos na região Neotropical. In: BARROSBATTESTI, D.M.; ARZUA, M.; BECHARA, G.H. (Org.). Carrapatos de Importância Médico-Veterinária da Região Neotropical: um guia ilustrado para a identificação de espécies. $1^{\mathrm{a}}$ ed. São Paulo: Vox/ICTTD-3/Butantan, 2006. p. 155-163.

LEITE, R.C.; OLIVEIRA, P.R.; CUNHA, A.P.; BELLO, A.C.P.P. Controle de ectoparasitos de eqüinos Amblyomma cajennense e Anocentor nitens. In: CONGRESSO BRASILEIRO DE PARASITOLOGIA VETERINÁRIA, 14 e SIMPÓSIO LATINO-AMERICANO DE RICKETTSIOSES, 2, 2006, Ribeirão Preto. Resumos... Jaboticabal: CBPV,. 2006. p. 120-124.

MARGOLIS, L.; ESCH, G.W.; HOLMES, J.C.; KURIS, A.M.; SCHAD, G.A. The use of ecological terms in parasitology (Report of an Ad Hoc Committee of the American Society of Parasitologist). Journal of Parasitology, v. 68, n. 1, p. 131-133, 1982.

MARTINS, J.R.; MEDRI, I.M.; OLIVEIRA, C.M.; GUGLIELMONE A. Ocorrência de carrapatos em tamanduá-bandeira (Myrmecophaga tridactyla) e tamanduá-mirim (Tamandua tetradactyla) na região do Pantanal Sul Mato-Grossense, Brasil. Ciência Rural, v. 34, n. 1, p. 293-295, 2004.

MEYER DE SCHAUENSEE, R. A guide to the birds of South America. Philadelphia: Academy of Natural Sciences of Philadelphia, 1982. 498 p.

MOISSANT DE ROMAN, E.; KLOBER, R.; MANZANILLA, J. Amblyomma cajennense (Fabricius, 1787) (Acari: Ixodidae) en los estados Aragua y Cojedes, Venezuela. Revista Cientifica, v. 12, n. 2, p. 94-96, 2002.

MOREIRA, J.A.; MAGALHÃES, O. Thypho exanthematico em Minas Gerais. Brasil Médico, v. 44, p. 465-470, 1935.

NAROSKY, T.; YZURIETA, D. Guia para la identificación de las aves de Argentina y Uruguay. $4^{\mathrm{a}}$ Ed. Buenos Aires: Asociación Ornitológica Del Plata, 1993. 340 p.

OJATSI, J. Estúdio biológico del chirigüe o capibara. Caracas: Fondo Nacional de Investigaciones Agropecuarias, 1973. 257 p.

OLIVEIRA, P.R. Amblyomma cajennense (Fabricius, 1787) (Acari: Ixodidae): Avaliação de técnicas para o estudo de dinâmica populacional e biotecnologia. 1998. 97 f. Tese (Doutorado em Ciência Animal) - Universidade Federal de Minas Gerais, Belo Horizonte, 1998.

ONOFRIO, V.C.; LABRUNA, M.B.; BARROS-BATTESTI, D.M. Comentários e chaves para as espécies do gênero Amblyomma. In: BARROS-BATTESTI, D.M.; ARZUA, M.; BECHARA G.H. (Org.). Carrapatos de Importância Médico-Veterinária da Região Neotropical: um guia ilustrado para a identificação de espécies. $1^{a}$ ed. São Paulo: Vox/ICTTD-3/Butantan, 2006. p. 553-113. 
PINTER, A.; VEIGA-BARREIROS, R.M.O.; TREVISAN, A.M.Y.; ALMEIDA, T.V.; NETO, E.J.R.; SOUZA, C.E.; LABRUNA, M.B. Relato de Amblyomma fuscum (Acari: Ixodidae) colhido em peridomicílio na cidade de Guarujá, estado de São Paulo. In: SIMPÓSIO BRASILEIRO DE ACAROLOGIA, 1, 2006, Viçosa. Anais... Viçosa: UFV, 2006. 1556 p.

PINTER, A.; DIAS, R.A.; GENNARI, S.M.; LABRUNA, M.B. Study of the seasonal dynamics, life cycle, and host specificity of Amblyomma aureolatum (Acari: Ixodidae), EUA. Journal of Medical Entomology, v. 41, n. 3, p. 324332, 2004.

PINTO, G.R.M. Contagem de fezes como índice de abundância de capivaras (Hydrochaeris hydrochaeris). 2003. $43 f$. Dissertação (Mestrado) - Escola Superior de Agricultura "Luiz de Queiroz", USP, Piracicaba, 2003.

ROHR, C.J. Estudo sobre Ixodidas no Brasil. Rio de Janeiro: Gomes \& Irmão, 1909. 220 p.

SANGIONI, L.A.; HORTA, M.C.; VIANNA, M.C.B.; GENNARI, S.M.; SOARES, R.M.; GALVÃO, M.A.M.; SCHUMAKER, T.T.S.; FERREIRA, F.; VIDOTTO, O.; LABRUNA, M.B. Rickettsial infections in animals and Brazilian spotted fever endemicity. Emerging Infectious Diseases., v. 11, n. 2, p. 265-270, 2005.

SERAFINI, P.S.; ANJOS, L.; ARZUA, M.; VOLPATO, G.; VARGAS, E.; POLLETTO, F. First Report of Ornithonyssus sylviarum (Acari: Macronyssidae) on Black Vulture (Coragyps atratus) nestlings from Brazil. Revista Brasileira de Parasitologia Veterinária, v.12, n.2, p. 9293, 2003.

SERRA-FREIRE, N.M.; PEIXOTO, B.T.M.; OLIVEIRA, V.L.; TEIXEIRA, R.H. Amblyomma nodosum Neumann, 1899: Contribuição ao estudo morfológico de machos e fêmeas. Revista Brasileira de Parasitologia Veterinária, v. 2, n. 2, p.105-108, 1993.
SICK, H. Ornitologia Brasileira. Edição revista e ampliada por José Fernando Pacheco. Rio de Janeiro: Nova Fronteira, 1997. $912 \mathrm{p}$.

SOUZA, D.G.S. Todas as aves do Brasil. Guia de Campo para Identificação. Feira de Santana: DALL, 1998. 350 p.

SOUZA, C.E.; CALIC, S.B.; CAMARGO, M.C.G.O. O papel das capivaras Hydrochaeris hydrochaeris na cadeia epidemiológica da Febre Maculosa Brasileira. Revista Brasileira de Parasitologia Veterinária, v. 13, (Supl. 1), p. 203-205, 2004.

SOUZA, S.S.A.L. Ecologia e técnicas de amostragem de ixodídeos em áreas endêmicas para febre maculosa brasileira na região de Campinas, 2004. 118f. Dissertação (Mestrado) - Universidade de Campinas, Campinas, 2004.

SOUZA, S.S.A.L.; MORAES, G.J.; SOUZA, C.E. Distribuição e dinâmica sazonal da população de carrapatos (Acari: Ixodidae) no campus da Escola Superior de Agricultura "Luiz de Queiroz" Piracicaba, SP. In: CONGRESSO BRASILEIRO DE PARASITOLOGIA VETERINÁRIA, 14 e SIMPÓSIO LATINO-AMERICANO DE RICKETTSIOSES, 2. 2006, Ribeirão Preto. Resumos... Jaboticabal: CBPV, 2006. p. 197

SUPERINTENDÊNCIA DE CONTROLE DE ENDEMIAS (SUCEN). Manual de Vigilância Acarológica. Superintendência de Controle de Endemias. São Paulo: Secretaria do Estado da Saúde. 2004. 62p.

SZABÓ, M.P.J.; CUNHA, M.; SANTOS A.P.; VICENTINI, F. Ticks (Acari: Ixodidae) associated with domestic dogs in Franca region, São Paulo, Brazil. Experimental and Applied Acarology, v. 969, n. 25, p. 290-293, 2001.

WILSON, J.G.; KINGER, D.R.; SAUER, J.R.; HAIR, J.A. Chemo-attraction in the lone star tick (Acarina: Ixodidae). I. Response of different developmental stages to carbon dioxide administered via traps. Journal of Medical Entomology, v. 9, n. 3, p. 245-252, 1972.

Recebido em 24 de agosto de 2007.

Aceito para publicação em 21 de outubro de 2008. 